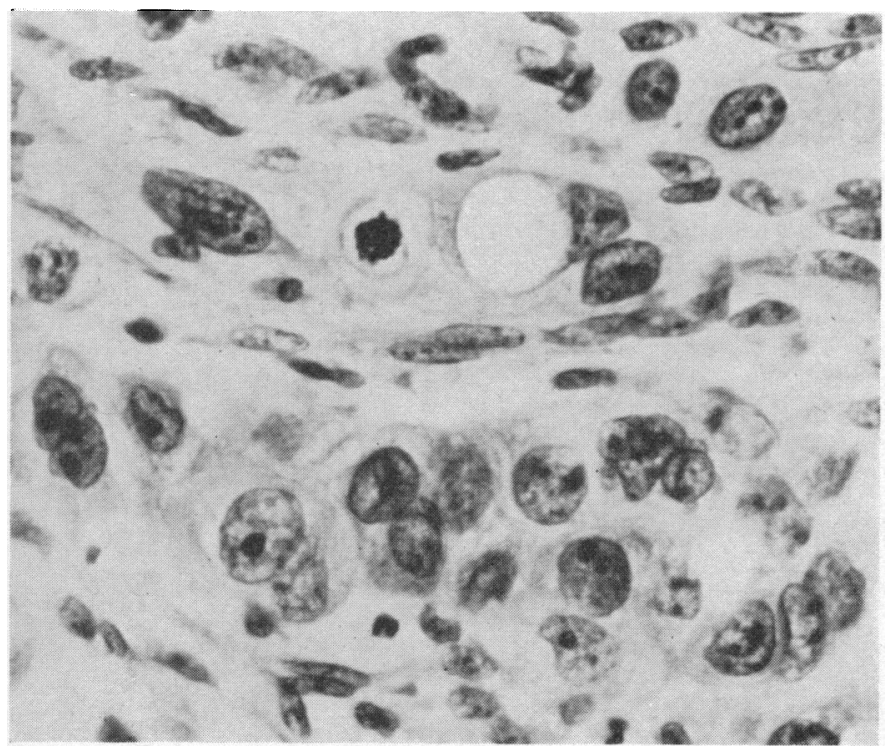

FIG. 2.-Foci of same tumour as in Fig. 1 containing viable tumour cells with scanty cytoplasm, relatively large nuclei with prominent nucleoli and a distinct chromatin pattern ; an apparently normal mitosis is present.

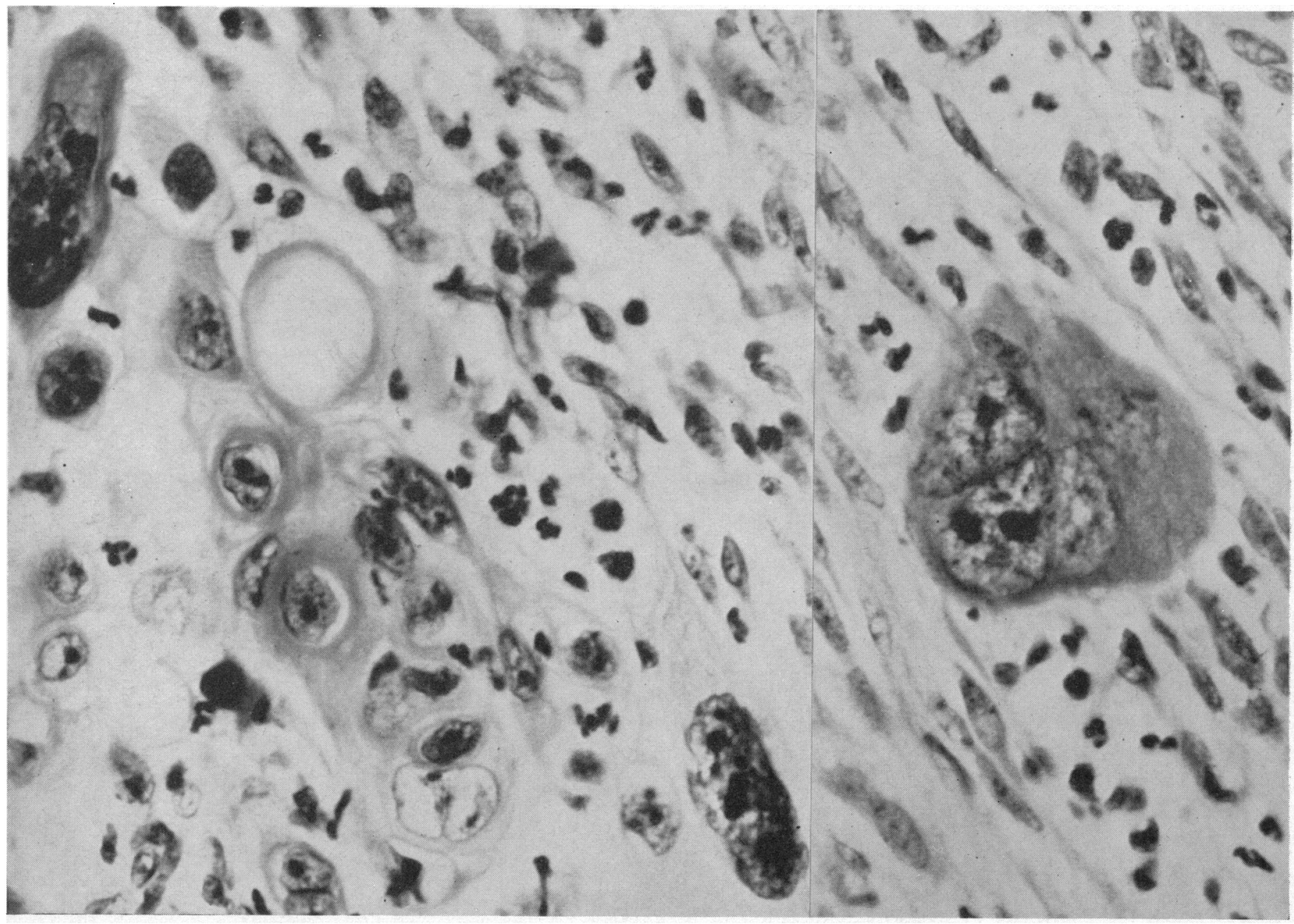

Fig. 1.-Section from an irradiated tumour showing bizarre non-viable cells in the form of two mononucleate monster cells and a multinucleate tumour giant cell, some degenerating cells, and keratinizing cells with condensation of the cytoplasm and well-defined cell walls. (H. and $E$.

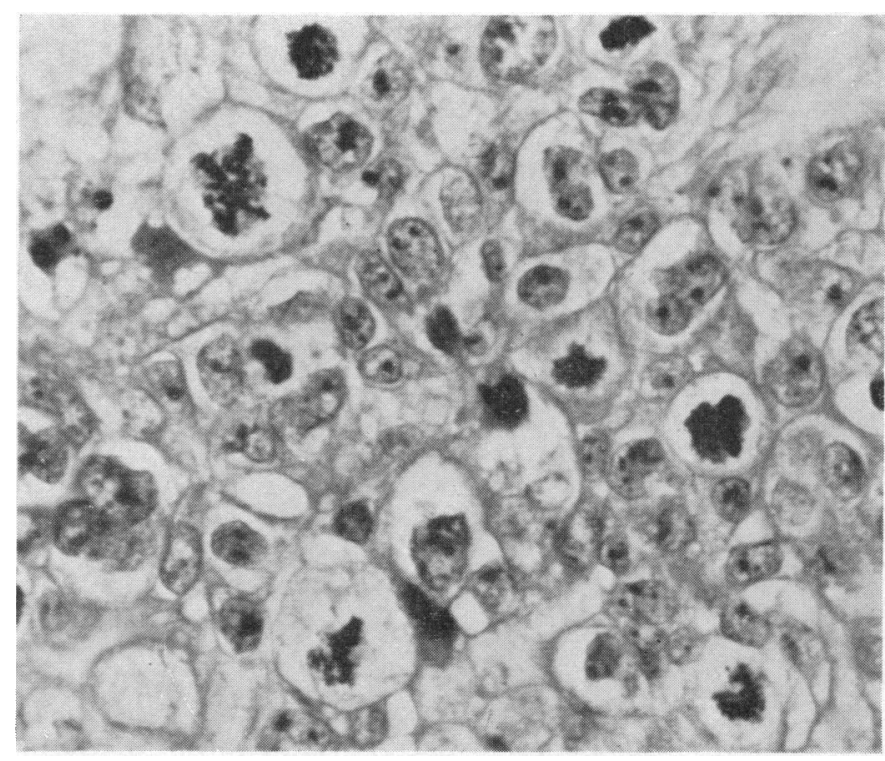

FIG. 3.-Section from an irradiated tumour showing small viable cells, degenerations, and bizarre abnormal mitotic figures. (H. and E. $\times 625$.)

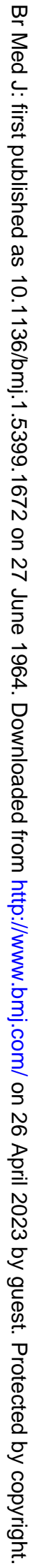
$(\mathrm{H}$. and E. $\times 625$.) 
a

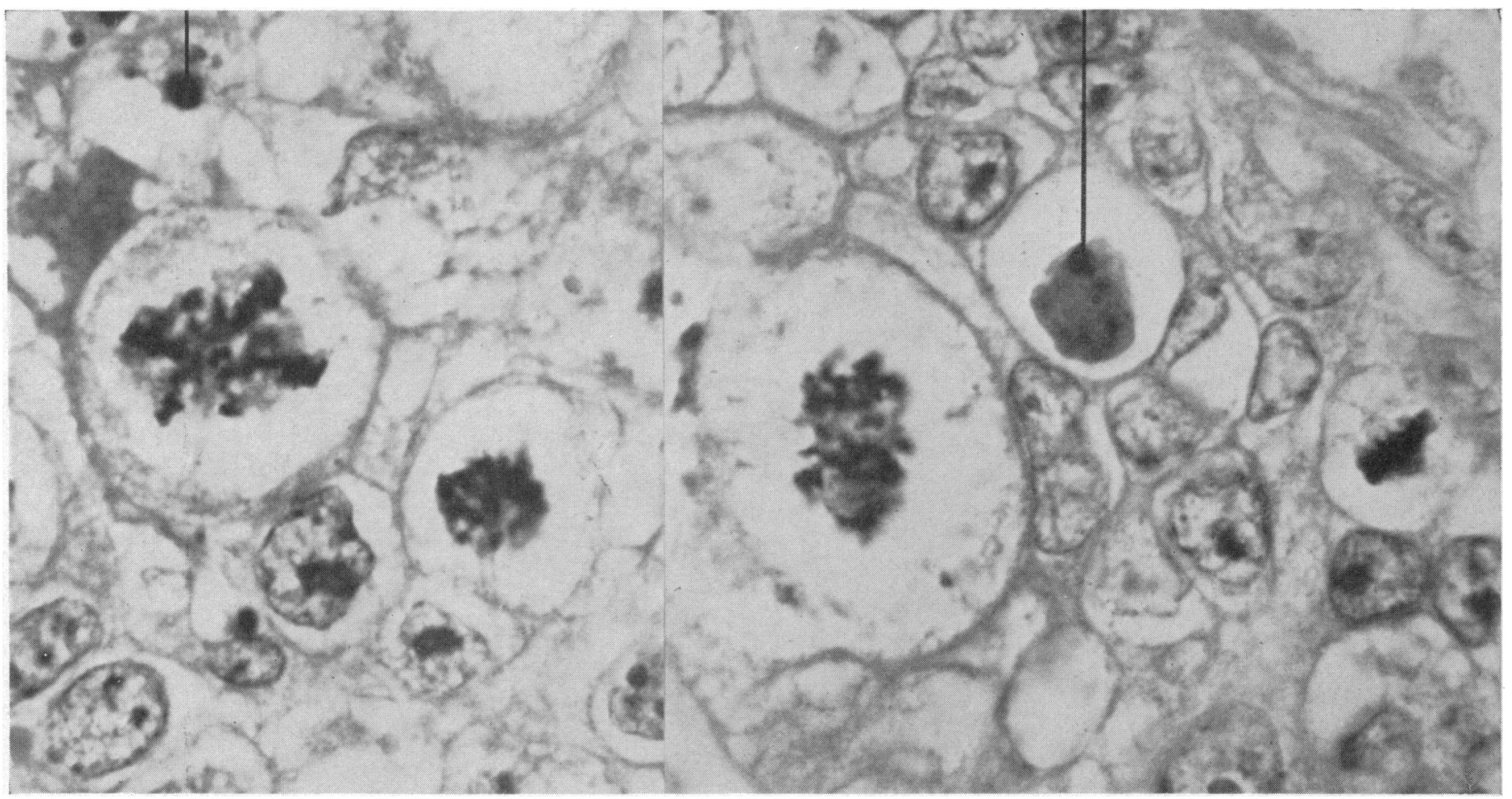

FIG. 4.-Showing some of the abnormal mitoses seen in Fig. 3 at higher magnification. Two degenerate cells (a) undergoing chromatolysis are also seen. (H. and E. $\times 2,340$.)

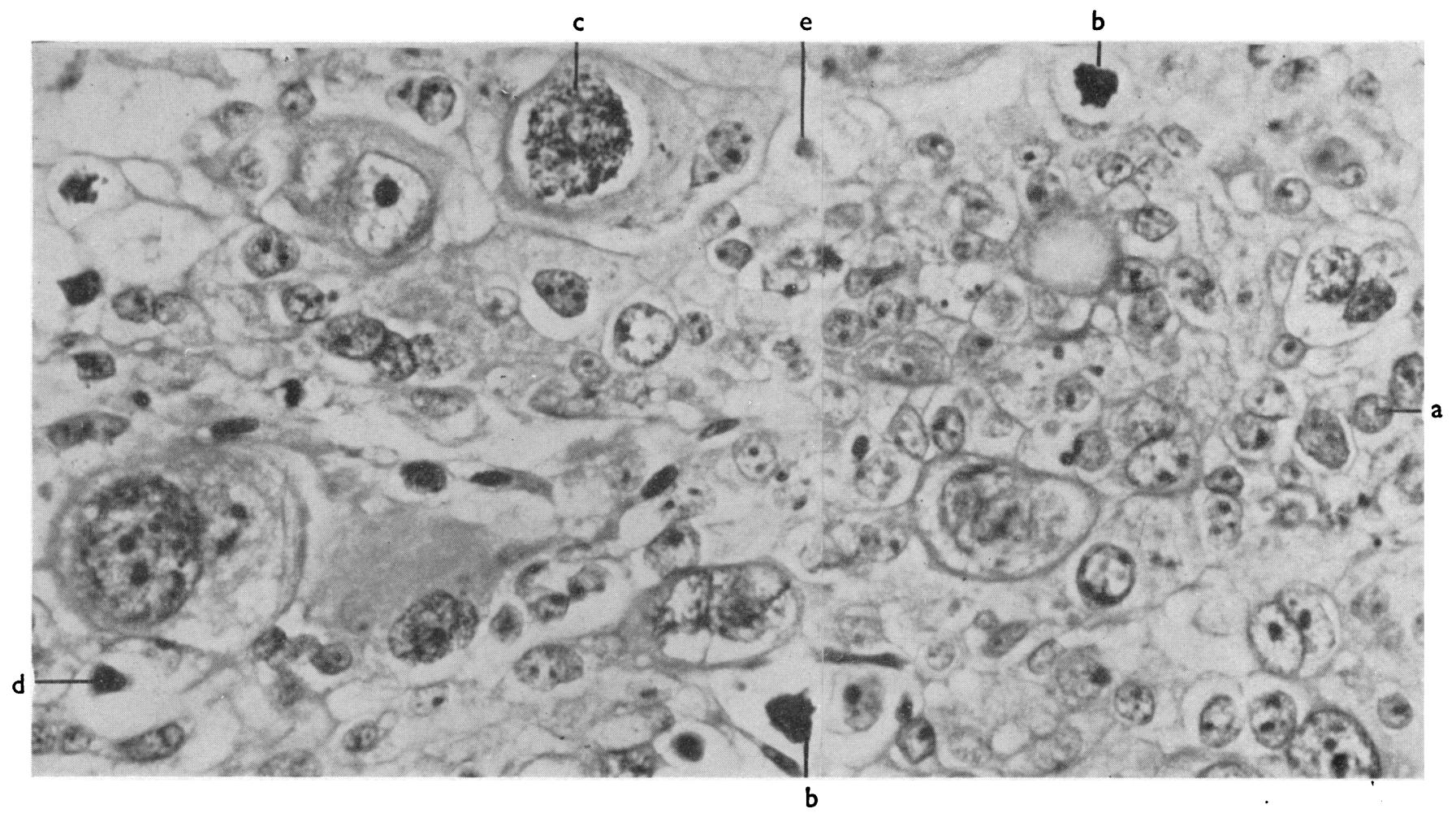

FIG. 5.- Section from same irradiated tumour as Figs. 3 and 4, showing numerous viable cells with scanty cytoplasm and intact nuclei (a); abnormal mitoses with clumped $(b)$ or scattered $(c)$ chromosomes; degenerating cells in the stage of pyknosis $(d)$; and chromatolysis $(e)$ and mononucleate and multinucleate monster cells. (H. and $\mathrm{E}$. $\times 645$.) 


\title{
Assessment of Response of Cervical Cancers to Irradiation by Routine Histological Methods
}

\author{
L. WALTER,* M.B., B.S., D.M.R.E. ; C. V. HARRISON, $†$ M.D. \\ A. GLUCKSMANN, $\ddagger$ M.D. ; C. P. CHERRY, $\ddagger$ M.B., CH.B.
}

[With Special Plate]

Brit. med. F., 1964, 1, 1673-1675

The purpose of this paper is to show that valuable prognostic information can be obtained from ordinary routine examination of serial biopsies taken from patients undergoing radiotherapy for cancer of the uterine cervix. The original method of examining serial biopsies from tumours before and during radiotherapy was devised in order to determine whether the early histological changes induced by irradiation could serve as a means of assessing the radiocurability of the individual tumour (Glucksmann, 1941). Since this research project was started it has been shown repeatedly that such a histological method gives a reliable indication of the local response to radiotherapy of the tumour in individual patients (Glucksmann and Spear, 1945 ; Glucksmann, 1946, 1947, 1952, 1958, 1961 ; Glucksmann and Way; 1948 ; Wood and Boag, 1950; McGarrity and Garvan, 1961 ; Merrill, Wood, and Zippen, 1961). In the early descriptions of the method cell counts in selected comparable areas of serial biopsies were advocated. It has since been proved that cell counts are unnecessary and that a modified and simplified form of the original technique gives as reliable an assessment of the local radiation response.

\section{Procedure}

A full clinical examination of the patient is made, and the extent of the tumour is assessed as accurately as possible, so determining the clinical stage. A provisional plan of treatment is decided on, but this may be modified when the patient is examined under an anaesthetic, and a further digital and visual examination of the cervix can be supplemented by cystoscopy.

In the series to be discussed in this paper the treatment consisted of three applications of radium, either alone or supplemented by $x$-ray therapy. In deciding on the exact plan for any one patient, the extent of the local disease, the type of tumour, and the age and general condition of the patient were all taken into consideration.

Whether the patient has had a biopsy for diagnosis or not, a pre-radiation biopsy is taken under an anaesthetic at the time of the first radium insertion, and this one is used by the pathologist for comparison with the later biopsies. Further specimens are obtained at the time of subsequent radium applications, again under anaesthetic. In the series under discussion the interval between the first and second biopsies was a week, and that between the second and third varied from one to three weeks. If a technique of treatment using one or two radium applications is used a biopsy should be taken a week after completion of the radium treatment, and if necessary another after a further week. These later biopsies

* Consultant Radiotherapist, Hammersmith Hospital, London. † Professor of Morbid Anatomy, Postgraduate Medical School, University of London.

¥ Working with a grant from the British Empire Cancer Campaign at the Strangeways Research Laboratory, Cambridge. can be taken without an anaesthetic if the cervix is exposed in a good light.

To avoid the possibility of misunderstanding it is necessary for the clinician and the pathologist to have agreed on the criteria to be used in assessing the response to radiation. These criteria are discussed later.

In the method recommended the pathologist has first to classify the histological type of the tumour before irradiation. Secondly, in biopsies taken during and after irradiation he has to note certain cytological changes and to make direct comparisons between biopsies taken before and after radiotherapy.

A prognosis based on radiation changes seen in serial biopsies can be given only if the dose and the time interval between beginning radiotherapy and the taking of the biopsies is known. The pathologist in all probability will not be given these facts; nor is it necessary, as the ultimate interpretation of the changes is in the hands of the clinician, who will have all these details.

\section{Technical Procedure}

In order to determine the effect of the radiation on the tumour it is essential for biopsy samples to include growing tumour foci. These are found at the margin of the tumour, where the blood supply is adequate, and the biopsy should be taken from this area to include the growing edge of the tumour and a margin of the adjacent tissue.

If the growth is of a proliferative type an adequate sample can be obtained with a biopsy punch, but if it is of the infiltrating ulcerative type it is better to cut a wedge with a knife. The specimen should be placed in fixative immediately.

The extent of the disease and the exact site of the preradiation biopsy should be carefully recorded (reference to the clock face is a convenient way of doing this), and subsequent biopsies should be taken from the neighbouring area, again taking the margin of the lesion. Necrotic tissue from the centre of the tumour or the surface of an ulcer is useless in evaluating the radiation response.

For the assessment of radiation changes in serial biopsies it is essential to have well-fixed material. Formol-saline should be avoided as it gives unsatisfactory cytological detail, and fixation in Susa solution (three hours is adequate for the average-sized biopsy followed by transfer to $80 \%$ alcohol) gives excellent results, and it is simple for the hospital staff to use as it is supplied in a form ready for immediate use. Lendrum's fixative (mercuric chloride in commercial formalin) also is simple to use and gives very good cytological detail. It may have an advantage over Susa solution as overfixation does not occur and specimens can be left for 24 hours in the fixative. This may be more convenient for the hospital staff. Our own specimens have all been fixed in one or other of these two fixatives. 
Fixation is followed by dehydration in alcohol in the usual way and then by routine paraffin-embedding. Haematoxylin and eosin staining of sections is quite adequate for assessing radiation changes.

\section{Essentials for the Pathological Report}

\section{Histological Classification of Tumour}

For practical purposes cervical cancers can be divided into three groups: (1) squamous carcinomas, including every gradation from the highly keratinized type to those in which keratinization is minimal and limited to a few cells ; (2) adenocarcinomas of varying grades of differentiation but all showing at least some mucin production; and (3) anaplastic tumours showing no sign of either keratin or mucin production and usually of anaplastic or even bizarre cytology.

\section{Criteria to be Reported}

To enable the clinician to interpret the prognosis the pathologist's report must contain information on the three following points :

Presence of Viable Tumour Cells.-Cells that are degenerating or proceeding to keratinization (Special Plate, Figs. 1 and 5) are of no importance. It is the viable cells that must be recognized. In ordinary haematoxylin and eosin sections these cells have a scanty basophilic cytoplasm and a relatively large and often hyperchromatic nucleus which may be dark-staining but with the nuclear structures distinct (Special Plate, Figs. 5 and 2). It is not necessary to count the number of viable cells present in sections of the postradiation biopsy, but the report must state whether there are many, few, only occasional, or no viable cells present.

Presence of Normal-looking Mitoses.-Bizarre and abnormal mitotic figures (Special Plate, Figs. 3 and 4) have a striking appearance and usually catch the eye. They can be disregarded as they lead to cell death and therefore are not of importance for the interpretation of the prognosis. On the other hand, it is essential for the clinician to know whether or not normal-looking mitoses are present in the post-radiation sections.

Perceptible Difference in Degree and Extent of Differentiation Between the Pre- and Post-radiation Sections.-This is the least important criterion, especially if much viable tumour persists for a week or more after beginning radiotherapy. However, in tumours of squamous type a marked increase in the degree and extent of keratinization between pre-radiation and the first post-radiation specimen, and between the latter and a second post-radiation biopsy, can be a guide in assessing prognosis. The same applies to increases in the amount of mucin and the number of mucin-producing cells in irradiated adenocarcinomas. Some of the tumours appearing initially anaplastic may show some keratinization or mucin production and occasionally both activities after irradiation.

In the presence of viable cells it is not relevant to mention whether an inflammatory reaction, necrosis, foreign-body giant cells, or keratinized debris are seen in the post-radiation sections. In the absence of any viable tumour such findings may indicate a good response to treatment, and thus can be of help to the clinician.

\section{Interpretation of Pathological Findings}

The prognosis based on the response to irradiation refers, obviously, to the local disease only. The final prognosis must depend on the distant spread as well.
An unfavourable response to treatment is indicated if the first post-radiation biopsy contains many viable tumour cells, and especially if there are normal-looking mitoses. The occurrence of only a minor increase in differentiation is also evidence of a poor response but is of less importance. The presence of large or bizarre cells is of no real prognostic value.

A favourable response to treatment is indicated when, in the first post-radiation biopsy, normal-looking mitoses in tumour cells cannot be found and when viable tumour cells are scanty. An increase in the degree and extent of keratinization between the first and second post-radiation biopsies is also a good sign.

In a minority of cases the radiation responses may have to be recorded as doubtful. Sometimes the post-radiation biopsy contains no recognizable tumour but only necrotic debris or inflammatory exudate. Sometimes there may be tumour present, but its response may be intermediate between the good and bad responses described above.

\section{Correlation Between Histological Prognosis and Five-year Results of Treatment}

In a series of 210 cases of carcinoma of the uterine cervix in clinical stages 1-4 treated by Dr. L. Walter at Hammersmith Hospital a pre-radiation biopsy and one or two additional biopsies taken during therapy were available and were submitted for assessment of the radiation effect. Information on radiation dose and the time interval between a given dose and the biopsy excision was supplied to the Strangeways Research Laboratory, Cambridge, cell counts were done on the material, and the prognosis was based on the quantitative analysis. The same material was submitted to the Pathology Department of the Postgraduate Medical School, Hammersmith, and was reported on routinely without information on the dose of radiation or the time in relation to radiation treatment of the biopsy excision. The reports on the post-radiation specimens specified whether or not viable tumour cells and normal-looking mitoses were present and whether or not there was an increase in differentiation of the tumour tissue as compared with the pre-radiation biopsy. The reports were sent to Dr. L. Walter, who judged the prognosis in the following way: if seven days after beginning radium treatment many viable cells and normal-looking mitoses were reported the prognosis was considered to be unfavourable; the absence of mitoses and the predominance of differentiated or degenerating tumour cells was taken as an indication of a favourable response to treatment.

At both centres no definite prognosis could be given in 105 cases. In the majority of instances this was because the postradiation samples contained no tumour tissue or, less often, because of ambiguous cellular changes. In the analysis these cases were regarded as having a doubtful prognosis.

Of the other 105 cases with a definite prognosis, $23 \%$ werc favourable and 77\% unfavourable according to the Postgraduate Medical School, while the corresponding figures for the Strangeways Research Laboratory were $12 \%$ and $88 \%$ respectively.

The Table compares the clinical result in the 210 cases with the prognosis given at the Postgraduate Medical School and at the Strangeways Research Laboratory. It shows that for both centres the five-year survival rate for cases given a

\begin{tabular}{|c|c|c|c|c|c|}
\hline \multirow{2}{*}{ Prognosis } & & \multicolumn{2}{|c|}{ Postgraduate Med. Sch. } & \multicolumn{2}{|c|}{ Strangeways Res. Lab. } \\
\hline & & No. of Cases & $\%$ & No. of Cases & $\%$ \\
\hline $\begin{array}{l}\text { Favourable } \\
\text { Unfavourable } \\
\text { Doubtful }\end{array}$ & $\begin{array}{l}\ldots \\
\cdots\end{array}$ & $\begin{array}{r}24 \\
81 \\
105\end{array}$ & $\begin{array}{l}80 \pm 9 \cdot 3 \\
30 \pm 5 \cdot 1 \\
50 \pm 4 \cdot 9\end{array}$ & $\begin{array}{r}12 \\
93 \\
105\end{array}$ & $\begin{array}{l}92 \pm 7 \cdot 8 \\
25 \pm 4 \cdot 5 \\
58 \pm 4 \cdot 8\end{array}$ \\
\hline
\end{tabular}


favourable prognosis is significantly higher than for those with an unfavourable prognosis, while that for patients in whom a definite prognosis could not be given is intermediate between the other two groups. Similar differences in survival rates between the three groups are seen if an analysis is made for clinical stage 1 and 2 cases separately and for stages 3 and 4 combined. (See histogram based on the data obtained at the Postgraduate Medical School.)

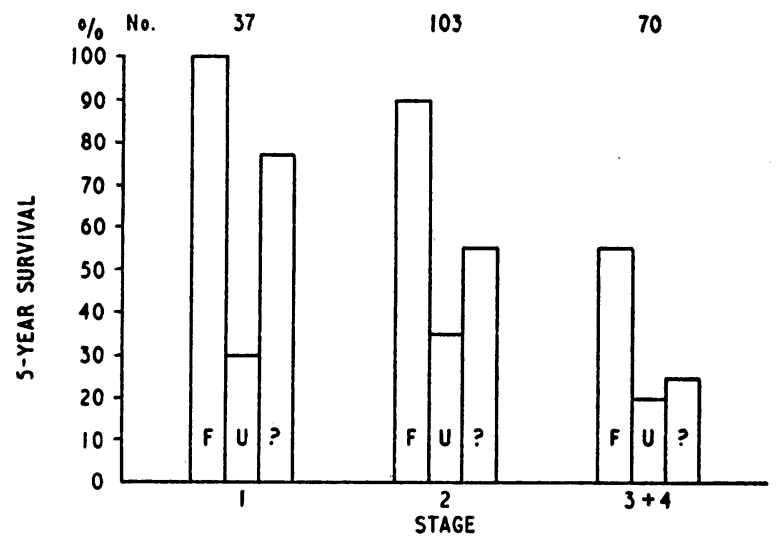

Histogram showing the relation of prognosis to five-year survival for clinical stages 1,2 , and 3 plus 4 . $\mathrm{F}=$ Favourable. U=Unfavourable. ? = Doubtful. No. $=$ Number of cases.

An overall survival rate of about $50 \%$ in the cases with a doubtful prognosis (see Table) suggests that the absence of tumour tissue or ambiguous changes in the post-radiation specimen did reflect a radio-curable tumour in about half of these patients. In some of these cases it might have been possible to give a definite prognosis if a second post-radiation biopsy had always been available. This is borne out in a series of 700 cases treated by $\mathrm{Mr}$. S. Way in Newcastle in which a second post-radiation biopsy reduced the incidence of doubtful prognoses to $12 \%$.

As can be seen from the Table the correlation between the prognosis and the five-year survival rates are very similar for the two methods used in assessing the response to radiation, and the differences in the favourable and in the unfavourable cases between the two centres are not statistically significant. In eight cases the prognosis given at the two centres was in complete disagreement; the Postgraduate Medical School gave a favourable prognosis in seven and an unfavourable prognosis in one, while the reverse was given at the Strangeways Research Laboratory. In four cases each centre gave the correct prognosis. In a further five cases the Postgraduate Medical School gave a favourable prognosis while the response was considered doubtful at the Strangeways Research
Laboratory. In another five cases the prognosis was regarded as unfavourable at the Strangeways Research Laboratory and doubtful at the Postgraduate School.

The high degree of agreement between prognoses based on a quantitative analysis and those based on pathological reports of radiation changes seen in serial biopsies shows that the histological assessment need not be a laborious procedure and is well within the scope of a routine pathology department. The fact that at the Postgraduate Medical School four different pathologists shared the reporting on a rota and yet achieved results comparable with those from a single research investigator indicates that the method outlined is easily reproducible.

The striking differences in the clinical result for cases with an unfavourable and a favourable prognosis indicate that the assessment of radiation changes in serial biopsies can be of use to the therapist in deciding on additional treatment in individual patients.

\section{Summary}

The response of cancers of the uterine cervix undergoing treatment by irradiation can be assessed by comparing biopsies taken before and during the course of treatment. This has previously been regarded as a research technique, but by making certain simple observations it is possible to apply the method in ordinary routine practice. The techniques necessary are described and a comparison is made of the findings on the same group of 210 patients by research workers and by a department carrying out routine reporting. The comparison of the histological reports with each other and with the clinical results after five or more years indicates that valid results can be obtained by a routine department.

\section{REPERENCES}

Glucksmann, A. (1941). Brit. F. Radiol., 14, 187.

(1946). Brit. med. Bull., 4, 26-30.

(1947). In Recent Advances in Clinical Pathology, edited by S. C Dyke, pp. 338-349. Churchill, London.

(1952). Brt. 7. Radiol., 25, 38.

(1958). Acta Un. int. Cancr., 14, 358.

(1961). IXth International Congress of Radiology, pp. 660-669. Thieme, Stuttgart.

and Spear, F. G. (1945). Brit. \%. Radiol., 18, 313.

- and Way, S. (1948). Y. Obstet. Gynaec. Brit. Emp., 55, 573.

McGarrity, K. A., and Garvan, J. M. (1961). In Radiobiology, edited by P. L. T. Ilbery, pp. 250-269. Butterworth, London.

Merrill, J. A., Wood, D. A., and Zippen, C. (1961). Proceedings of Conference on Research on the Radiotherapy of Cancer, 1960, pp. 113-121. Amer. Cancer Soc., New York.

Wood, C. A. P., and Boag, J. W. (1950). Researches on the Radiotherapy of Oral Cancer. Spec. Rep. Ser. med. Res. Coun. (Lond.), No. 267. H.M.S.O., London. 